\title{
Enhanced spontaneous emission from InAs/GaAs self-organized quantum dots in a GaAs photonic-crystal-based microcavity
}

\author{
Peichen Yu and Pallab Bhattacharya ${ }^{a)}$ \\ Solid State Electronics Laboratory, Department of Electrical Engineering and Computer Science, \\ The University of Michigan, Ann Arbor, Michigan 48109-2122 \\ Jui-Ching Cheng \\ Department of Electronics Engineering, Chang Gung University, Taoyuan, Taiwan, Republic of China
}

(Received 3 December 2002; accepted 19 February 2003)

\begin{abstract}
Spontaneous emission characteristics of self-organized InAs/GaAs quantum dots embedded in a photonic-crystal-based microcavity are theoretically investigated. Results are obtained from a three-dimensional quasi-mode analysis of the photonic-crystal microcavity and through calculations of energy levels and eigenstates for electrons and holes in self-organized quantum dots. The spontaneous emission rate from quantum dots is significantly enhanced by up to a factor of 14 , compared to that in free space. In addition, the maximum coupling efficiency to the fundamental mode is close to unity. (C) 2003 American Institute of Physics. [DOI: 10.1063/1.1566470]
\end{abstract}

\section{INTRODUCTION}

The quantum dot microcavity light emitter has emerged as a promising candidate for single photon light sources. ${ }^{1-4}$ In particular, the analysis of spontaneous emission characteristics of self-organized quantum dots (QDs) in photonic crystal (PC) microcavities has not been fully accomplished because of two reasons. First, the three-dimensional (3D) mode analysis of a PC cavity is nontrivial due to the complexity of the cavity geometry. Although several groups ${ }^{5,6}$ have reported 3D simulations of the spontaneous emission characteristics of an ideal dipole in PC microcavities, using finitedifference time-domain (FDTD) methods, a more comprehensive description of cavity modes is yet to appear. Second, interactions between self-organized QDs and cavity modes cannot be fully described without an explicit calculation of the transition matrix elements, which in turn requires complete analyses of the cavity modes of a 3D cavity and the eigenstates of the quantum dots. In this article we report the calculated spontaneous emission characteristics of InAs/ GaAs self-organized QDs embedded in a PC microcavity.

\section{THEORETICAL MODEL}

To calculate the enhancement factor of the QD spontaneous emission and the coupling efficiency to the desired modes, we have developed a 3D quasi-mode analysis for PC microcavities. A description of the mode density, including the guided modes, Bragg resonant modes, and radiation modes (or so-called quasi-modes), in wave-vector (k) space is obtained. In addition, the eigenstates for electrons and holes in a GaAs-capped, pyramidal, and coherently strained InAs/GaAs QD have been calculated by an eight-band $\mathbf{k} \cdot \mathbf{p}$ method. The calculation of the cavity modes and the eigen-

${ }^{a)}$ Electronic mail: pkb@eecs.umich.edu states of the dots allows us to take the modified mode density into account and directly calculate the transition matrix element in the Fermi golden rule.

The device design is schematically shown in Fig. 1. The defect microcavity is surrounded in the transverse direction by a two-dimensional (2D) triangular-lattice photonic crystal and vertically by a pair of Bragg mirrors. Accordingly, the device can be approximated as a combination of a longitudinal cavity and a transverse cavity. ${ }^{7}$ The longitudinal cavity is a $\lambda$-thick GaAs spacer sandwiched by 10.5 -pair AlAs/GaAs Bragg mirrors on both sides. The transverse cavity is a missing defect in a $5 \times 5$ triangular lattice photonic crystal of air holes. This device configuration supports vertically and horizontally resonant modes. It may be noted that if the reflectivity of the Bragg mirrors is large enough, the device will perform as a vertical-cavity surface-emitting laser (VCSEL) with photonic crystal (PC) lateral confinement (the PC-VCSEL) $;{ }^{8}$ otherwise, it behaves as a resonant microcavity light emitting diode.

For mode analysis, we assume that the transverse properties of the cavity modes are determined by the photonic crystal, whereas the Bragg mirrors only determine the field profile in the longitudinal $z$ direction. In other words, we ignore the field coupling outside the cavity, which is generally a good approximation for tightly confined modes above cutoff conditions. The eigenmodes of the longitudinal and transverse cavities are computed using standard techniques. ${ }^{9}$ The band structures $\omega_{k_{z}}^{1}\left(k_{\rho}\right)$ and $\omega_{k_{\rho}}^{t}\left(k_{z}\right)$ for the longitudinal and transverse cavities are computed independently, where $k_{\rho}$ is the wave vector in the $k_{x}-k_{y}$ plane, and then plotted in $\mathbf{k}$ space using the dispersion relation, $\omega=c|\mathbf{k}|$, where $|\mathbf{k}|$ $=\sqrt{k_{x}^{2}+k_{y}^{2}+k_{z}^{2}}$. Owing to the nonuniform distribution of modes per unit wave vector, we refer to such a representation of the band structure as the mode density. A 3D cavity mode is defined as the cross point of a longitudinal band and a transverse band in $\mathbf{k}$ space. As a result, this mode should satisfy longitudinal and transverse boundary conditions si- 


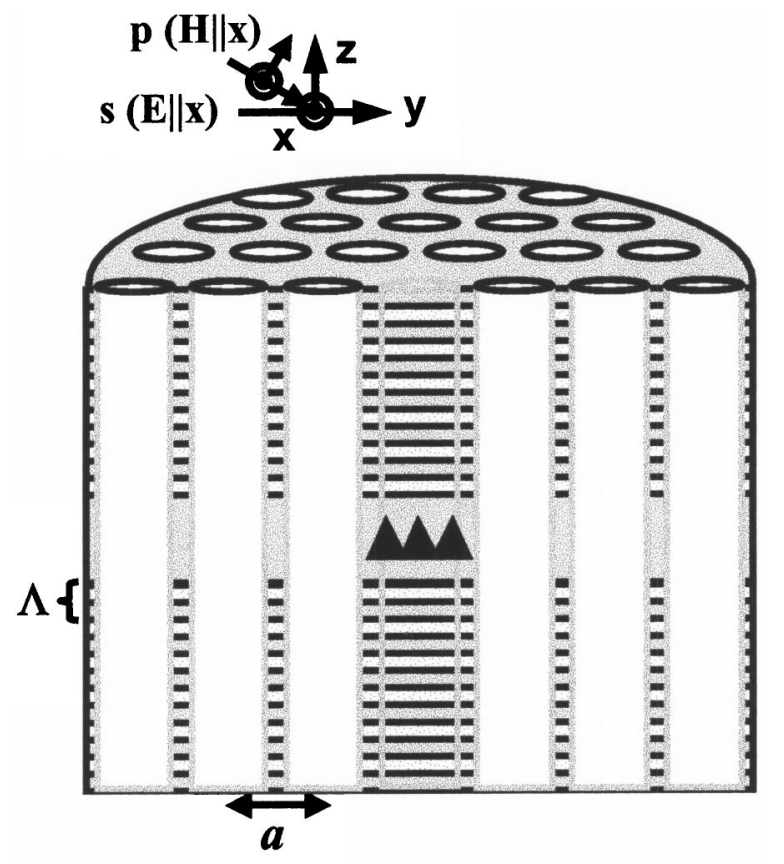

FIG. 1. The device schematic, which consists of InAs/GaAs self-organized quantum dots as the active medium embedded in a photonic crystal defect microcavity. The two-dimensional photonic crystal consists of a triangular lattice of air holes. In the vertical direction, the microcavity is surrounded by GaAs/AlAs Bragg mirrors.

multaneously. The field pattern of the confined 3D cavity modes can be determined as a scalar product of the longitudinal field and the transverse field components, given that their polarizations are identical in the cavity region.

\section{RESULTS AND DISCUSSION}

Figures 2(a)-2(c) show the calculated transverse and longitudinal mode densities. The cavity modes can be characterized as the Bragg resonant mode (solid line), guided mode (dashed line), and a number of radiation modes (shaded region). There exists a clear stop band in the longitudinal mode densities, but not in the transverse cavity. As seen in Fig. 2(c), the stop band and Bragg resonant mode vanish at the Brewster angle for $p$ polarization. By choosing a proper periodicity ratio, $a / \Lambda$, where $a$ and $\Lambda$ are, respectively, the periodicities of the triangular lattice of air holes and the Bragg mirrors, the mode distribution of a 3D cavity in $k$ space can be obtained, as shown in Fig. 3. Two modes in this figure are of particular interest, defined as the vertical and horizontal fundamental modes. The vertical fundamental mode is a product of the Bragg resonant mode of the longitudinal cavity and the guided mode of the transverse cavity, which propagates and resonates vertically. For a small Bragg mirror loss, as in a VCSEL, this mode becomes the dominant lasing mode. The horizontal fundamental mode is created by the Bragg resonant mode of the transverse cavity and the guided mode of the longitudinal cavity. This mode resonates and propagates in transverse directions. Here, the photonic crystal functions as a $2 \mathrm{D}$ dielectric mirror and the Bragg mirror acts as a multilayer waveguide. As seen in Fig. 3, the wavelength of both modes is blueshifted from the Bragg

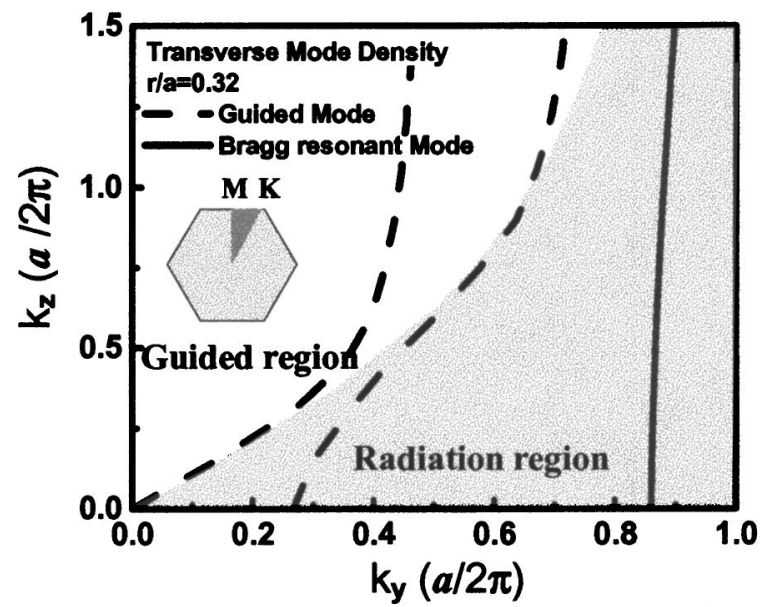

(a)

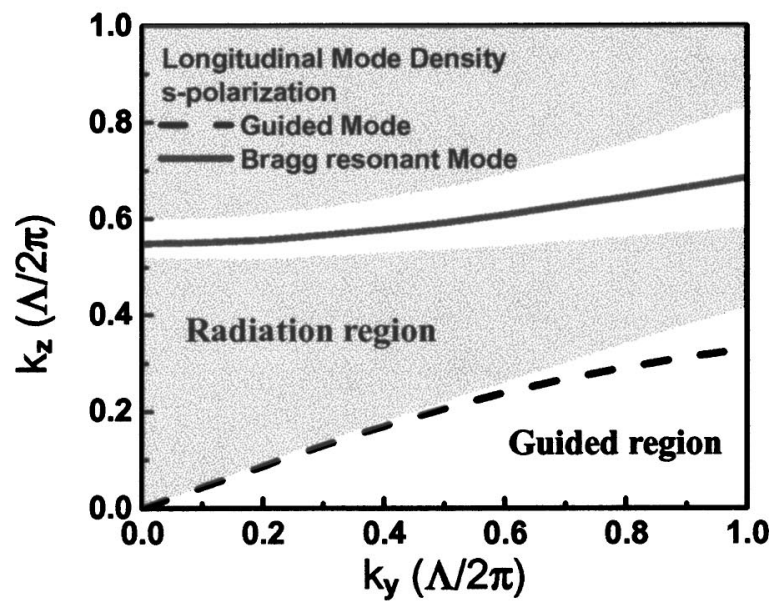

(b)

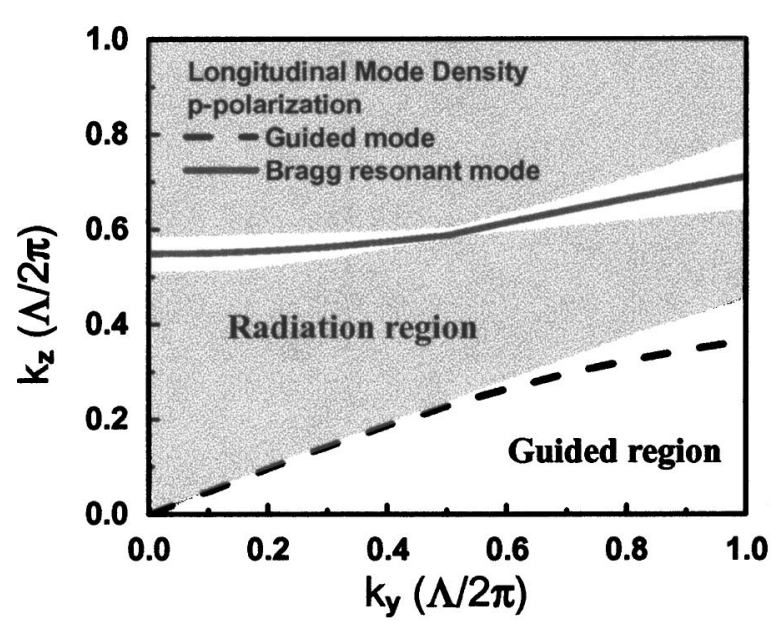

(c)

FIG. 2. (a) Calculated transverse mode density along the $\Gamma-M$ direction for a 2D photonic crystal defect cavity with a triangular lattice of air holes. The wave vectors are normalized with respect to the lattice constant, $a$. The calculated longitudinal mode densities are shown in (b) for $s$ polarization and in (c) for $p$ polarization. The wave vectors are normalized with respect to the periodicity of the Bragg mirrors, $\Lambda$. 


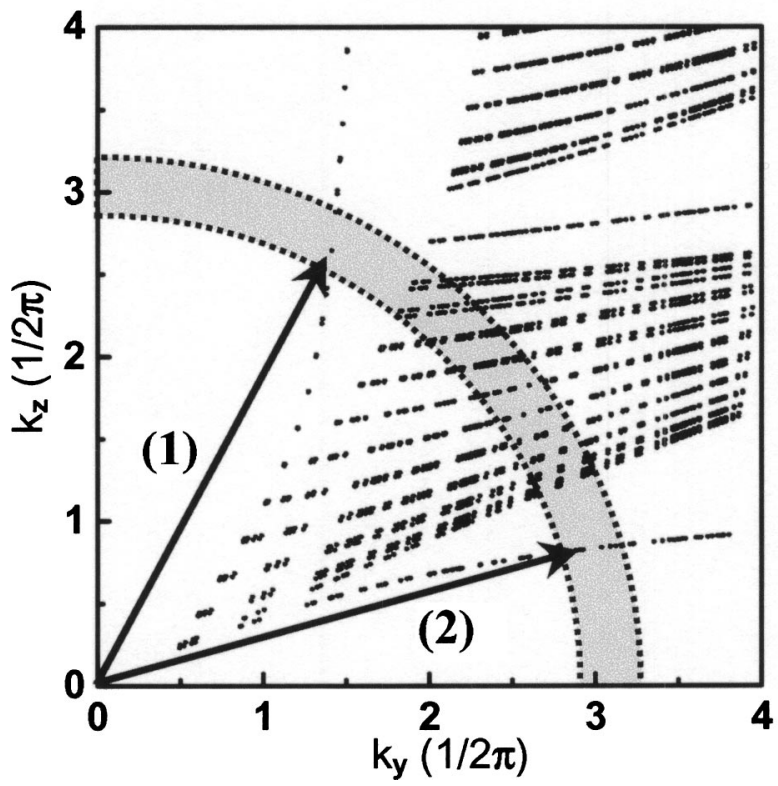

FIG. 3. Three-dimensional mode density with design parameters $\lambda_{0}$ $=1.2 \mu \mathrm{m}, \Lambda=0.213 \mu \mathrm{m}$, and $a=0.302 \mu \mathrm{m}$ for (1) the vertical fundamental mode and (2) the horizontal fundamental mode. The quarter circular shell shows the emission spectrum from the active QD region around the fundamental modes. This region indicates that light emits and couples to the modes that overlap with the emission spectrum of the active region.

wavelength due to their lateral mode confinement. The shaded quarter circular shell represents the emission spectrum from the active region and indicates that light emits and couples to the modes that overlap with the emission spectrum of the active region.

Self-assembled InAs quantum dots are formed on GaAs as coherently strained islands when the lattice mismatch exceeds $1.8 \% .{ }^{10}$ In our simulation, we consider a GaAs-capped, pyramidal quantum dot with $\{101\}$ facets and a square base in the (001) plane. ${ }^{11}$ The strain tensor is calculated using the valence force field model of Keating ${ }^{12}$ and Martin. ${ }^{13}$ An eight-band $\mathbf{k} \cdot \mathbf{p}$ formulation, which includes coupling between conduction and valence bands, is used for determining the bound states in a dot. ${ }^{14,15}$ Within the dipole approximation, the transition matrix element, $\left|M_{f i}\right|^{2}$, for a single dot can be expressed as ${ }^{14}$

$$
\begin{aligned}
\left|M_{f i}\right|^{2} & =\left|\left\langle\Psi_{f}\left|\widetilde{A}_{k} \vec{\varepsilon} \cdot \vec{p}\right| \Psi_{i}\right\rangle\right|^{2} \\
& =\left|\left\langle\sum_{i=1}^{8} u_{i} \phi_{i}^{(f)}\left|\widetilde{A}_{k} \vec{\varepsilon} \cdot \vec{p}\right| \sum_{j=1}^{8} u_{j} \phi_{j}^{(i)}\right\rangle\right|^{2} \\
& \approx\left(\widetilde{A}_{k, x} F_{f i}^{x}+\hat{A}_{k, y} F_{f i}^{y}+\widetilde{A}_{k, z} F_{f i}^{z}\right) P_{c v}^{2},
\end{aligned}
$$

where $\widetilde{A}_{k}$ denotes the averaged electric field amplitude of the mode $k$ in the active region, $\vec{p}$ is the momentum matrix element, $\vec{\varepsilon}$ is the unit polarization vector, $u_{i}$ denote the eight Bloch functions, $\phi_{i}$ the corresponding envelope functions, $F_{f i}$ are the envelope function overlap components, and $P_{c v}$ is a scalar optical matrix element. The spontaneous emission rate in the cavity can then be expressed, using the Fermi golden rule, as

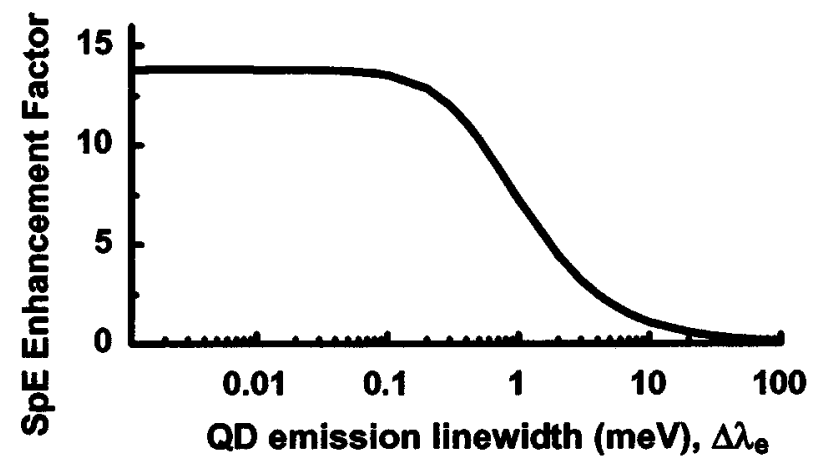

(a)

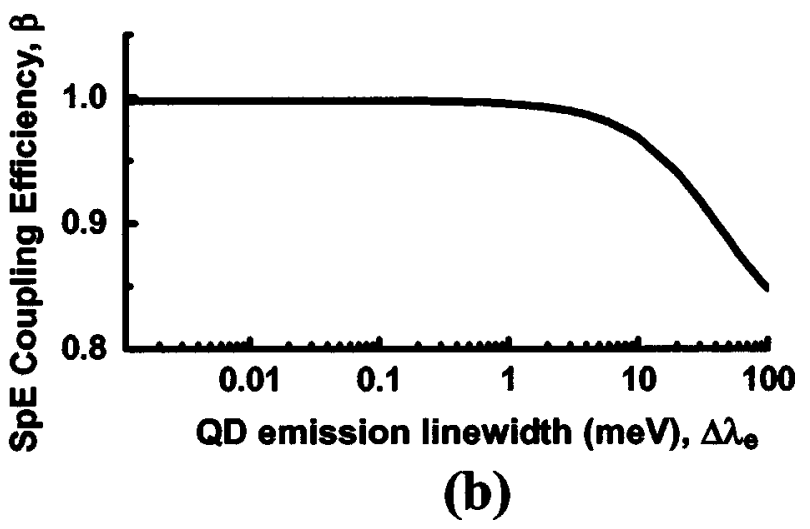

FIG. 4. Calculated values of (a) spontaneous emission enhancement factor, and (b) coupling factor, $\beta$, as a function of QD emission linewidth, $\Delta \lambda_{e}$. Maximum enhancement factor is $\sim 14$ and coupling efficiency is $\sim 99 \%$. The calculations have been done for a cavity of thickness $\lambda$ and with values $r / a=0.32, \lambda_{e}=\lambda_{c}=1.2 \mu \mathrm{m}$, and $\Delta \lambda_{c}=1 \mathrm{meV}$. When the QD emission spectrum completely overlaps the cavity linewidth, $\Delta \lambda_{c}$, i.e., $\Delta \lambda_{e} \ll \Delta \lambda_{c}$, both factors can no longer be increased.

$$
\begin{aligned}
\frac{1}{\tau_{s p}}= & \frac{2 \pi}{\hbar} \int \sum_{k} \frac{e^{2}}{m_{0}^{2} \omega_{k}^{2}}\left|M_{f i}\right|^{2} \rho_{c}\left(E-E_{k}\right)_{E_{k}=\hbar \omega_{k}} \\
& \times \rho_{e}\left(E-E_{i j}\right) f^{e}\left(E_{i}^{e}\right) f^{h}\left(E_{j}^{h}\right) d E,
\end{aligned}
$$

where $\rho_{c}$ is a normalized Lorentzian with a finite linewidth to take into account the optical loss for cavity modes. $\rho_{e}$ is a normalized Gaussian centered at a possible transition $E_{i j}$ between $i, j$ states in the dot. The linewidth of this Gaussian represents the inhomogeneous broadening of the emission from the QD ensemble due to size variation of the dots occurring during the self-organization process. The linewidth therefore represents the number of dots in the cavity. $f^{e}$ and $f^{h}$ are the occupancy of electrons and holes, respectively.

For an InAs QD with a square base of $160 \AA$ and a height of $80 \AA$, the emission wavelength is found to be 1.2 $\mu \mathrm{m}$, both from theory ${ }^{15}$ and experiment. ${ }^{16}$ It is assumed that the ground state emission from QDs is spectrally aligned to the fundamental modes. In addition, the $\mathrm{Q}$ factors for both the vertical and horizontal fundamental modes are taken to be $\sim 1000,{ }^{7,17}$ calculated by the radiation loss in the vertical and horizontal directions. Hence the cavity linewidth, $\Delta \lambda_{c}$, is $\sim 1 \mathrm{meV}$. The spontaneous emission rate of each cavity mode can then be calculated and summed to obtain the total spontaneous emission rate in the cavity, using Eq. (2). The calculated spontaneous emission enhancement factors are 
plotted in Fig. 4(a) as a function of the QD emission linewidth, $\Delta \lambda_{e}$. The enhancement factor is the ratio of the total spontaneous emission rate in the cavity to that in free space. As seen in Fig. 4(a), the enhancement factor saturates when $\Delta \lambda_{e} \ll \Delta \lambda_{c}$. Under this condition, the spectrum of the fundamental modes completely overlaps the QD emission spectrum and further enhancement of the spontaneous emission rate is no longer possible. The maximum enhancement factor is $\sim 14$.

The calculated coupling efficiency of spontaneous emission to the fundamental modes, $\beta$, is plotted in Fig. 4(b). $\beta$ is the ratio of the spontaneous emission rates of two fundamental cavity modes, vertical and horizontal, to the total spontaneous emission rate of all cavity modes. The maximum coupling efficiency is $\sim 99 \%$. In Fig. 4(a), the saturation region of the enhancement factor corresponds to the conditions under which the Purcell factor is derived. The Purcell factor describes the amount by which the spontaneous emission rate is enhanced for an emitter in resonance with a cavity mode. We compare our results with the Purcell factor, ${ }^{18} F_{p}$ $=3 \lambda^{3} Q /\left(4 \pi^{2} V_{m}\right)$. The mode volume $V_{m}$ of a 3D fundamental mode is estimated to be $5 \lambda^{3}$, which gives $F_{p}=15$. It suggests that the Purcell factor can predict the enhanced spontaneous emission rate for an ideal system where only a single or a few dots are embedded in the microcavity, i.e., $\lambda_{e}=\lambda_{c}$ and $\Delta \lambda_{e} \ll \Delta \lambda_{c}$. Otherwise, the enhancement of the spontaneous emission can deteriorate very quickly when the QD emission linewidth becomes much broader than the cavity linewidth, as in the case of high density or multiple layers of self-organized QDs enclosed in the microcavity.

\section{ACKNOWLEDGMENTS}

Discussions with Professor Jasprit Singh and Dr. Boaz Kochman are gratefully acknowledged. The work is being supported by the Army Research Office under Grant No. DAAD 19-01-1-0527.

${ }^{1}$ O. Benson, C. Santori, M. Pelton, and Y. Yamamoto, Phys. Rev. Lett. 84, 2513 (2000).

${ }^{2}$ P. Michler, A. Kiraz, C. Becher, W. V. Schoenfeld, P. M. Petroff, L. Zhang, E. Hu, and A. Imamoğlu, Science 290, 2282 (2000).

${ }^{3}$ C. Santori, M. Pleton, G. Solomon, Y. Dale, and Y. Yamamoto, Phys. Rev. Lett. 86, 1502 (2001).

${ }^{4}$ Z. Yuan, B. Kardynal, R. M. Stevenson, A. J. Shields, C. J. Lobo, K. Cooper, N. S. Beattie, D. A. Ritchie, and M. Pepper, Science 295, 102 (2002)

${ }^{5}$ X. Yu, J. Vučković, R. Lee, O. Painter, A. Scherer, and A. Yariv, J. Opt. Soc. Am. B 16, 465 (1999).

${ }^{6}$ J. Hwang, H. Ryu, and Y. Lee, Phys. Rev. B 60, 4688 (1999).

${ }^{7}$ T. Baba, T. Hamano, F. Koyama, and K. Iga, IEEE J. Quantum Electron. 27, 1347 (1991).

${ }^{8}$ D. S. Song, S. H. Kim, H. G. Park, C. K. Kim, and Y. H. Lee, Appl. Phys. Lett. 80, 3901 (2002).

${ }^{9}$ S. G. Johnson and J. D. Joannopoulos, Opt. Express 83, 173 (2001)

${ }^{10}$ P. R. Berger, K. Chang, P. Bhattacharya, J. Singh, and K. K. Bajaj, Appl. Phys. Lett. 53, 684 (1988).

${ }^{11}$ D. Leonard, M. Krishnamurthy, C. M. Reaves, S. P. Denbaars, and P. M. Petroff, Appl. Phys. Lett. 63, 3203 (1993).

${ }^{12}$ P. N. Keating, Phys. Rev. 145, 637 (1966).

${ }^{13}$ R. M. Martin, Phys. Rev. B 1, 4005 (1970).

${ }^{14}$ H. Jiang and J. Singh, Physica E (Amsterdam) 2, 614 (1998); 2, 720 (1998).

${ }^{15}$ H. Jiang and J. Singh, IEEE J. Quantum Electron. 34, 1188 (1998).

${ }^{16}$ S. Ruvimov, P. Werner, K. Scheerschmidt, U. Gösele, J. Heydenreich, U. Richter, N. N. Ledentsov, M. Grundmann, D. Bimberg, M. Ustinov, A. Yu. Egorov, P. S. Kop'ev, and Zh. I. Alferov, Phys. Rev. B 51, 14766 (1995).

${ }^{17}$ C. Kim, W. J. Kim, A. S. Stapleton, J.-R. Cao, J. D. O'Brien, and P. D. Dapkus, J. Opt. Soc. Am. B 19, 1777 (2002).

${ }^{18}$ J.-M. Gérard and B. Gayral, J. Lightwave Technol. 17, 2089 (1999). 